\section{Research Square}

Preprints are preliminary reports that have not undergone peer review.

They should not be considered conclusive, used to inform clinical practice, or referenced by the media as validated information.

\title{
Modeling the Cost of Management Options for small benign non- functional adrenal incidentaloma: A Decision Tree Analysis
}

\author{
Wenjun Ni \\ Zhuhai City People's Hospital \\ Xingxing Chen \\ Zhuhai City People's Hospital \\ Xiao Ye \\ Zhuhai City People's Hospital \\ Guofu Pang \\ Zhuhai City People's Hospital \\ Yan Liu \\ Zhuhai City People's Hospital
}

Xiaoxu Yuan ( $\square$ chnk200210ydp@163.com)

Zhuhai People's Hospital https://orcid.org/0000-0001-5194-6224

\section{Research}

Keywords: small benign non-functional adrenal incidentaloma, surgery, surveillance, economic analysis

Posted Date: January 18th, 2021

DOI: https://doi.org/10.21203/rs.3.rs-144432/v1

License: (c) (i) This work is licensed under a Creative Commons Attribution 4.0 International License. Read Full License 


\section{Abstract}

Background Adrenal incidentaloma (Al) is the term for any unsuspected lesion of the adrenal gland found by radiologic imaging, usually done for other indications. Although it is consistently recommended that hyper-functional tumor and suspicious malignancy need surgery, the management of patients with small benign non-functional adrenal incidentaloma remains controversial. Here we develop a detailed mathematical decision analysis model comparing the projected management costs for small benign non-functional adrenal incidentaloma.

Methods A detailed model was developed that integrates projected costs for a few possible treatment outcomes. Starting values and probabilities were derived from a comprehensive meta-analysis of 1298 patients. Hypothesis testing was performed using sensitivity analysis.

Results The model predicts a cost premium for follow-up compared with surgery. The tornado analysis identify the cost of follow-up for once as the most important variables for our model. One-way sensitivity analysis demonstrated that cost equivalence could be met with $¥ 4359.5$ for once follow-up, keeping other factors constant.

Conclusion Our models of treatment for small benign non-functional adrenal incidentaloma showed significant cost differences for follow-up vs immediate surgery. The cost of follow-up for once featured threshold values.

\section{Background}

Adrenal incidentaloma (Al) is the term for any unsuspected lesion of the adrenal gland found by radiologic imaging, usually done for other indications. Since Al is a clinical dilemma, several organizations including the National Institutes of Health (NIH), the European Society of Endocrinology ${ }^{1}$ (ESE) and the European Network for the Study of Adrenal Tumors (ENSAT) published guidelines to help in the evaluation and management of these patients. Although it is consistently recommended that hyper-functional tumor and suspicious malignancy need surgery, the management of patients with small benign non-functional adrenal incidentaloma remains controversial and some experts recommend surgery rather than surveillance ${ }^{2-4}$. Contrast to temporary guidelines, in our previous report ${ }^{5}$, silent malignancy and pheochromocytoma were not rare; also some studies demonstrated that follow-up was associated with higher incidence of metabolic syndrome $e^{6}$. As small benign non-functional adrenal incidentaloma within initial assessment may progress to hyper-functional, larger size or malignancy plus anxiety is burdensome for some patients, both in economic and psychology, surgery could be another choice besides followup.

Here we develop a detailed mathematical decision analysis model comparing the projected management costs for small benign nonfunctional adrenal incidentaloma. It is built on a meta-analysis ${ }^{7}$ and reflects a multi-institutional clinical experience. The model incorporates a variety of clinical outcomes downstream from the original therapy decision(Fig. 1), given that these events would potentially influence costs.

\section{Methods}

Decision analytic models are methods of estimating and calculating outcomes by identifying the clinical question, disaggregating the problem into discrete units to include all reasonable choices and consequences, and assigning probabilities and value to the various events and outcomes. The decision model was designed and analyzed using TreeAge Pro software with the Healthcare suite (available from: http://www.treeage.com). Follow-up VS surgery of small benign non-functional adrenal incidentaloma constituted the arms of the decision tree (Fig. 1).

\section{model assumptions}

According meta-analysis from Loh $\mathrm{HH}^{7}$, we assume $1.5 \%$ of cases end up surgery for developing Cushing's syndrome or pheochromocytoma at the third year of follow-up, and the same percentage of cases accept surgeries at the forth year. The rest of others complete the five-year follow-up without surgery.

\section{costs, charges, and time values}

The surgeon's fee and anesthesiologist's fee, as well as the charges of pre-operation stay, post-operation stay and follow-up, were all derived from Zhuhai Peoples' hospital of Guangdong province in China in 2018.( Table 1) 
Table 1

cost and charge of each patient

\begin{tabular}{|c|c|c|c|c|c|c|c|c|c|c|c|c|}
\hline PID & $\mathrm{TC}$ & LOS & DC & Presc & LOPreSS & DCBS & $\mathrm{AF}$ & SF & PostSC & LOPostSS & DCAS & TOS \\
\hline 485468 & 22712.2 & 11 & 2064.7 & 7092.2 & 6 & 1182.0 & 4836.2 & 8721.9 & 2061.9 & 5 & 412.4 & 110 \\
\hline 490273 & 47748.9 & 16 & 2984.3 & 6848.3 & 5 & 1369.7 & 10507.3 & 9889.5 & 20503.8 & 11 & 1864.0 & 265 \\
\hline 454471 & 31107.3 & 21 & 1481.3 & 11695.8 & 14 & 835.4 & 5048.0 & 10284.5 & 4079.0 & 7 & 582.7 & 185 \\
\hline 488745 & 26848.4 & 26 & 1032.6 & 11165.8 & 19 & 587.7 & 3063.2 & 7841.4 & 4778.1 & 7 & 682.6 & 115 \\
\hline 433868 & 29440.7 & 20 & 1472.0 & 8751.1 & 10 & 875.1 & 4503.5 & 8781.7 & 7404.4 & 10 & 740.4 & 255 \\
\hline 484723 & 33960.3 & 14 & 2425.7 & 10129.9 & 5 & 2026.0 & 3456.3 & 9842.4 & 10531.8 & 9 & 1170.2 & 135 \\
\hline 493007 & 18755.3 & 8 & 2344.4 & 6309.3 & 4 & 1577.3 & 2904.2 & 7982.6 & 1559.2 & 4 & 389.8 & 90 \\
\hline 493110 & 25618.5 & 11 & 2329.0 & 7979.7 & 5 & 1595.9 & 3079.6 & 9865.4 & 4693.8 & 6 & 782.3 & 240 \\
\hline 493721 & 20803.7 & 18 & 1155.8 & 9015.6 & 15 & 601.0 & 3289.5 & 6164.9 & 2333.7 & 777.9 & 120 & \\
\hline 491777 & 21885.4 & 14 & 1563.2 & 5757.8 & 9 & 639.8 & 2866.7 & 8185.4 & 5075.6 & 5 & 1015.1 & 120 \\
\hline 491791 & 24055.5 & 10 & 2405.6 & 7515.3 & 5 & 1503.1 & 4030.4 & 9435.1 & 3074.7 & 5 & 614.9 & 135 \\
\hline 491770 & 29463.2 & 10 & 2946.3 & 9714.9 & 3 & 3238.3 & 3471.9 & 9385.4 & 6891.0 & 7 & 984.4 & 145 \\
\hline 453794 & 23038.6 & 11 & 2094.4 & 5694.9 & 6 & 949.2 & 4516.8 & 9321.4 & 3505.5 & 5 & 701.1 & 110 \\
\hline 495208 & 22424.9 & 10 & 2242.5 & 7859.3 & 5 & 1571.9 & 2590.4 & 8185.4 & 3789.9 & 5 & 758.0 & 120 \\
\hline 350331 & 23169.5 & 13 & 1782.3 & 8085.7 & 8 & 1010.7 & 3953.9 & 8710.1 & 2419.8 & 5 & 484.0 & 300 \\
\hline 335045 & 20997.9 & 9 & 2333.1 & 5309.6 & 4 & 1327.4 & 2585.2 & 9345.4 & 3757.7 & 5 & 751.5 & 130 \\
\hline 491232 & 25399.1 & 13 & 1953.8 & 12666.1 & 8 & 1583.3 & 2662.5 & 8453.9 & 1616.7 & 5 & 323.3 & 256 \\
\hline 478221 & 21475.1 & 9 & 2386.1 & 5278.4 & 4 & 1319.6 & 3006.7 & 9821.6 & 3368.5 & 5 & 673.7 & 110 \\
\hline 497285 & 20564.4 & 11 & 1869.5 & 6913.5 & 7 & 987.6 & 3206.2 & 8713.9 & 1730.8 & 4 & 432.7 & 145 \\
\hline 494903 & 23263.7 & 10 & 2326.4 & 8873.5 & 6 & 1478.9 & 3006.5 & 9345.4 & 2038.3 & 4 & 509.6 & 120 \\
\hline 498139 & 21571.2 & 11 & 1961.0 & 3328.6 & 3 & 1109.5 & 3393.5 & 8673.9 & 6175.2 & 8 & 771.9 & 80 \\
\hline 393877 & 24408.7 & 11 & 2219.0 & 6807.0 & 6 & 1134.5 & 3549.0 & 10170.4 & 3882.3 & 5 & 776.5 & 145 \\
\hline 322924 & 21557.2 & 10 & 2155.7 & 5854.5 & 5 & 1170.9 & 2398.2 & 9833.4 & 3471.2 & 5 & 694.2 & 95 \\
\hline 477631 & 34762.2 & 10 & 3476.2 & 8842.4 & 3 & 2947.5 & 5087.0 & 9445.4 & 11387.5 & 7 & 1626.8 & 255 \\
\hline 499334 & 31729.9 & 18 & 1762.8 & 7410.6 & 6 & 1235.1 & 4906.3 & 11006.4 & 8406.6 & 12 & 700.6 & 230 \\
\hline 501786 & 22406.2 & 9 & 2489.6 & 7917.6 & 4 & 1979.4 & 3836.2 & 7596.4 & 3056.0 & 5 & 611.2 & 145 \\
\hline 501960 & 23369.9 & 14 & 1669.3 & 8412.1 & 9 & 934.7 & 5055.9 & 7019.7 & 2882.2 & 5 & 576.4 & 125 \\
\hline 497189 & 25976.5 & 11 & 2361.5 & 10443.5 & 5 & 2088.7 & 3294.4 & 9394.1 & 2844.5 & 6 & 474.1 & 135 \\
\hline 482067 & 23366.3 & 11 & 2124.2 & 7904.8 & 8 & 988.1 & 4657.9 & 9270.1 & 1533.5 & 3 & 511.2 & 135 \\
\hline 504594 & 29434.7 & 20 & 1471.7 & 10011.5 & 6 & 1668.6 & 4321.1 & 8113.1 & 6989.0 & 14 & 499.2 & 195 \\
\hline 507882 & 21273.9 & 12 & 1772.8 & 7704.4 & 8 & 963.0 & 3704.4 & 7570.4 & 2294.8 & 4 & 573.7 & 155 \\
\hline 508619 & 22370.2 & 14 & 1597.9 & 10125.4 & 10 & 1012.5 & 2727.7 & 7419.6 & 2097.4 & 4 & 524.4 & 115 \\
\hline 458474 & 20939.3 & 11 & 1903.6 & 6488.4 & 4 & 1622.1 & 3210.3 & 7249.7 & 3991.0 & 7 & 570.1 & 120 \\
\hline 506979 & 34178.0 & 11 & 3107.1 & 16712.4 & 7 & 2387.5 & 5769.1 & 8243.1 & 3453.3 & 4 & 863.3 & 105 \\
\hline
\end{tabular}


The costs for each treatment arm were calculated as the average costs for all patients in each arm and were cumulative from beginning until the end of follow-up or surgery.

Annual inflation rate is not constant, we incorporate approximate average number, $5 \%$, into cost.

\section{sensitivity analysis}

To address the effect of the individual variables on our model and test the validity of our model assumptions, we performed 1-way sensitivity analysis on all model parameters. This was performed by individually varying each variable for a range of values, keeping all other variables constant. If the resulting costs were unchanged, the analysis was considered "insensitive" to the tested variable. Otherwise, we calculated the threshold(ie, "crossover point") at which a given value for the variable in question would change which treatment arm featured the lowest overall costs.

The variables that featured threshold values on 1-way analysis were also used for 2-way sensitivity analysis, in which 2 variables were varied simultaneously, and the thresholds were measured.

\section{Results}

\section{base case analysis}

The base case analysis demonstrated follow-up featured the lowest overall cost per patient (¥7213) compared with immediate surgery (¥24691)(Fig. 2).

\section{sensitivity analysis}

The tornado analysis, which included 1-way sensitivity analysis of all parameters, was used to identify the most important variables for our model. The cost of follow-up for once was the most important, because varying this parameter during the sensitivity analysis resulted in the greatest effects on costs (wider bars in the tornado diagram); other values that had a minimal effect on the analysis(Fig. 3).

To identify cost of follow-up for once resulting in cost equivalence between follow-up and immediate surgery, we performed a one-way sensitivity analysis. This analysis demonstrated that cost equivalence could be met with $¥ 4359.5$ for once follow-up, keeping other factors constant(Fig. 4).

Because cost of follow-up for once was the only parameter demonstrating threshold value, we focused the 2-way sensitivity analysis on this set of parameters. Using two-way sensitivity analysis, cost of follow-up for once and length of post-surgery stay were varied to evaluate how these factors affected the choice of the least costly therapy option. For the majority of cost of follow-up for once and length of post-surgery stay, follow-up shows a clear cost advantage(Fig. 5).

\section{Discussion}

When facing small benign non-functional adrenal incidentaloma, patients and urologists must decide between follow-up and surgery. This decision hinges on the critical issues of effectiveness, morbidity, and cost. Both options have good outcomes. Only I Belmihoub ${ }^{8}$ repoted one

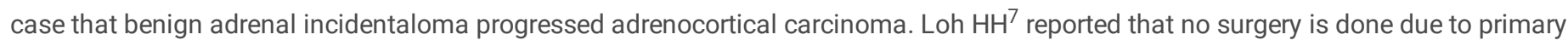
adrenal malignancy during follow-up. Likewise, the morbidity of surgery is mild ${ }^{9-12}$. Cost may, therefore, play an important role in directing clinical decisions in this disease. A detailed decision-tree model was developed for projecting the management costs for small benign nonfunctional adrenal incidentaloma. The model's advantage lies in the ease with which various hypotheses can be rapidly tested through the use of sensitivity analysis.

Enhanced recovery after surgery(ERAS) is popular nowadays, however, shortening length of stay(LOS) does not meet the equivalent point in our model. ERAS could not make immediate surgery less cost than follow-up.

The current model includes treatment-related morbidity, because the various cost derived from the actual charge from patients and/or medical insurance bureau. Three of 21 patients had surgery complication, which is similar with other reports ${ }^{9-12}$. One patient complicated major hemorrhage and transferred to open surgery, another one had urinary infection and fever, the third one had pneumonia after surgery. Although these three patients stayed longer than average length of stay and cost more than the others, they were discharged with good health finally.

It is important to differentiate the cost analysis presented here from a formal cost-effectiveness analysis. Given the small relative differences in mortality among follow-up and immediate surgery, use of mortality as a measure of effectiveness is unavailable. An alternative strategy 
would be to assign estimated quality-of-life utility values to all the outcome states within the model. As noted previously, the morbidity differences between follow-up and immediate surgery are also likely to be small. Estimates based on such small and controversial differences in morbidity would add an unacceptable degree of systematic error to results, and would cloud their interpretation. As more comprehensive morbidity data become available, however, extension of this analysis to include effectiveness would likely be valuable.

It is critical to appreciate the limitations as well as the strengths of a modeling exercise of this type. Any model depends on a set of critical assumptions, some of which can

be supported by the literature, whereas others are simply estimates or reflect local clinical practice. Cost modeling is also inherently dependent on institution and region, and

these results may not be valid for all institutions. Yet the structure of the model reflects general management principles for small benign nonfunctional adrenal incidentaloma, and should be easily translated to other centers provided that institution-specific cost components are modified.

Finally, because we examined the costs from the perspective of the medical establishment, indirect costs, such as psychological factor, were not included. No published work is available regarding the indirect costs of follow-up vs immediate surgery. This is an area worthy of additional study.

\section{Conclusions}

Our models about management of small benign non-functional adrenal incidentaloma showed significant cost differences for follow-up vs immediate surgery. The cost of follow-up for once featured threshold values.

\section{Declarations}

\section{Ethics approval and consent to participate}

Due to the retrospective nature of this study, ethics approval by our Institutional Review Board was obtained and guidelines on patient consent have been met.

\section{Consent for publication}

All authors have read final manuscript and consented for publication.

\section{Availability of data and materials}

The dataset supporting the conclusions of this article is included within the article and its additional files.

\section{Competing interests}

The authors declare that they have no competing interests.

\section{Funding}

There is no fund supporting this research.

\section{Authors' contributions}

WJN, XXC and XY were responsible for data collection and analysis, interpretation of the results, and writing the manuscript. GFP, YL and XXY were responsible for conducting the study design, data analysis and interpretation.

\section{Acknowledgements}

The authors of the article would like to thank and acknowledge Shan Qiu for her aid of collecting data.

\section{References}

1. Fassnacht M, Arlt W, Bancos I, et al. Management of adrenal incidentalomas: European Society of Endocrinology Clinical Practice Guideline in collaboration with the European Network for the Study of Adrenal Tumors. European Journal of Endocrinology. 
2016;175(2):G1-G34.

2. Celejewski K, Pogorzelski R, Toutounchi S, et al. Adrenal incidentaloma - diagnostic and treating problem - own experience. De Gruyter. 2018;13:281-284.

3. Morelli V, Scillitani A, Arosio M, Chiodini I. Follow-up of patients with adrenal incidentaloma, in accordance with the European society of endocrinology guidelines: Could we be safe? J Endocrinol Invest 2017;40:331-333.

4. Yeomans H, Calissendorff J, Volpe1 C, Falhammar H, Mannheimer B. Limited value of long-term biochemical follow-up in patients with adrenal incidentalomas-a retrospective cohort study. BMC Endocrine Disorders. 2015;15:1-6.

5. Ye Y-I, Yuan X-x, Chen M-k, Dai Y-p, Qin Z-k, Zheng F-f. Management of adrenal incidentaloma: the role of adrenalectomy may be underestimated. BMC Surgery. 2016;16(41).

6. Libe R, Dall'Asta C, Barbetta L, Baccarelli A, Beck-Peccoz P, Ambrosi B. Long-term follow-up study of patients with adrenal incidentalomas. European Journal of Endocrinology. 2002;147:6.

7. HH L, A Y, HS L. The natural progression and outcomes of adrenal incidentaloma: a systematic review and meta-analysis. Minerva Endocrinologica. 2017;42(1):77-87.

8. Belmihoub I, Silvera S, Sibony M, et al. From benign adrenal incidentaloma to adrenocortical carcinoma: an exceptional random event. European Journal of Endocrinology. 2017;176(6):K15-19.

9. Ari A, Buyukasik K, Tatar C, et al. Laparoscopic Treatment of Adrenal Tumors: A Single-Center Experience with 58 Patients. Surgery Research and Practice. 2016.

10. B Ö, A A, E E, et al. Laparoscopic surgery in functional and nonfunctional adrenal tumors: A singlecenter experience. Asian Journal of Surgery. 2016;39:137-143.

11. Öz B, Akcan A, Emek E, et al. Laparoscopic surgery in functional and nonfunctional adrenal tumors: A single-center experience. Asian Journal of Surgery. 2016;39(3):137-143.

12. Kang T, Gridley A, Richardson WS. Long-Term Outcomes of Laparoscopic Adrenalectomy for Adrenal Masses. Journal of Laparoendoscopic \& Advanced Surgical Techniques. 2015;25(3):182-186.

\section{Figures}

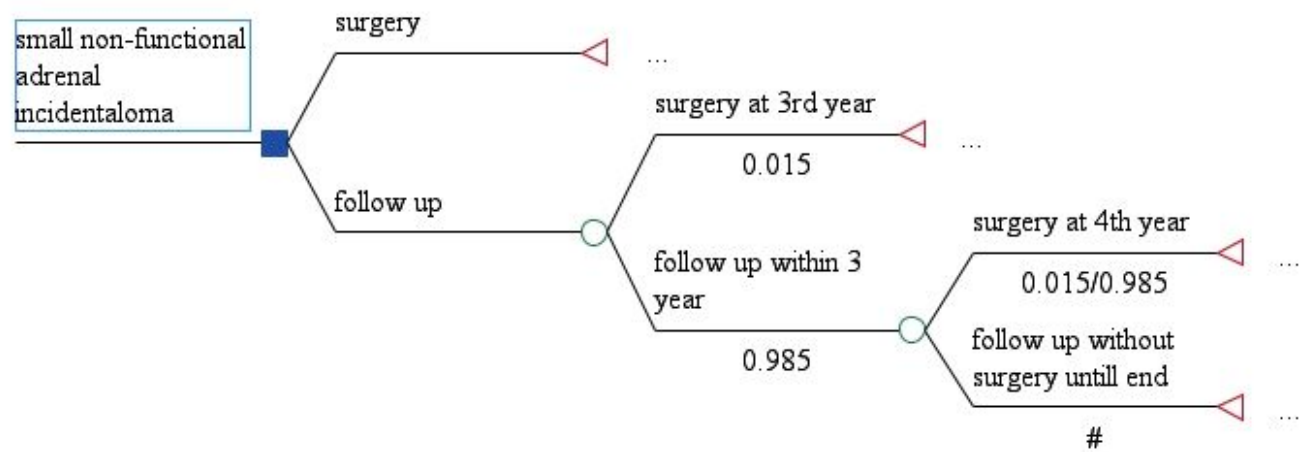

\section{Figure 1}

Basic diagram of the decision tree model for the cost of treatment options for small benign non-functional adrenal incidentaloma 


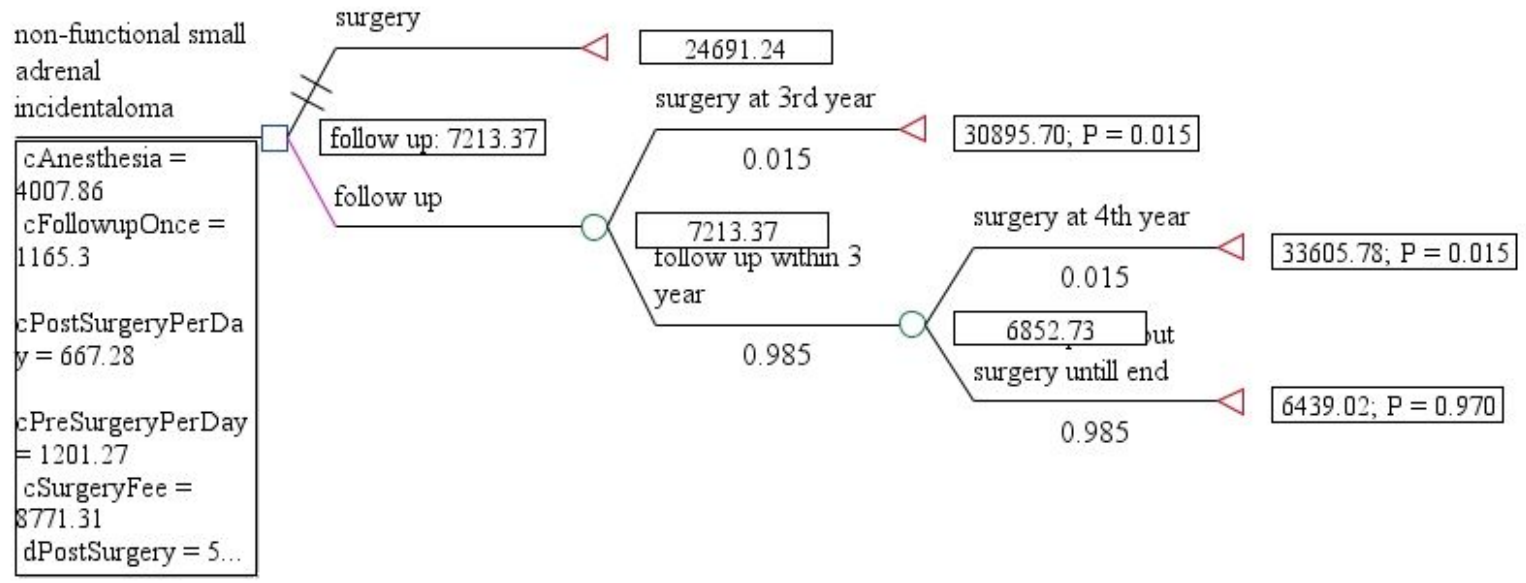

Figure 2

comparison of costs of 2 options Sensitivity Analysis

Tornado Sensitivity Analysis

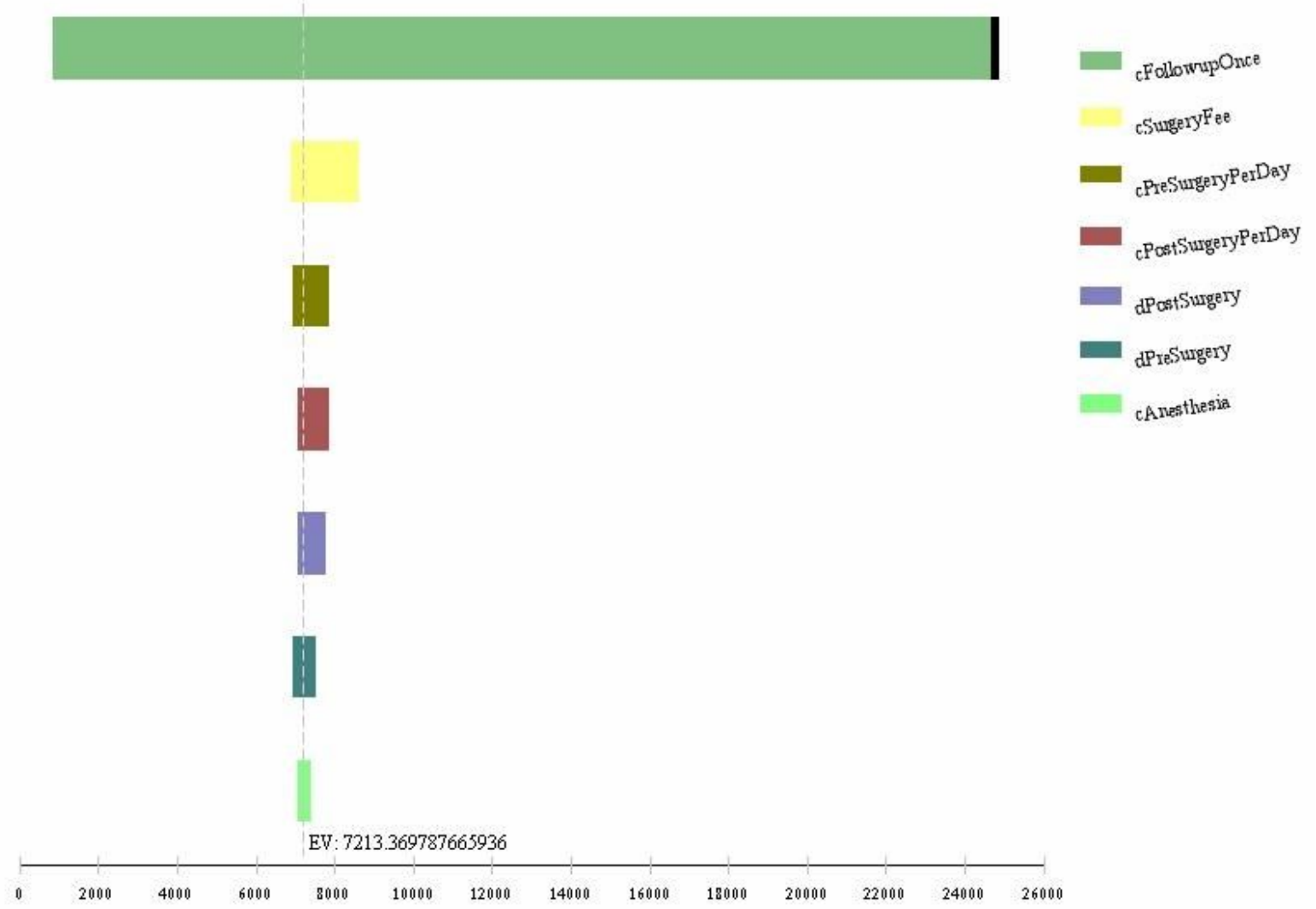

Figure 3

Tornado sensitivity analysis of our model 


\section{Sensitivity Analysis}

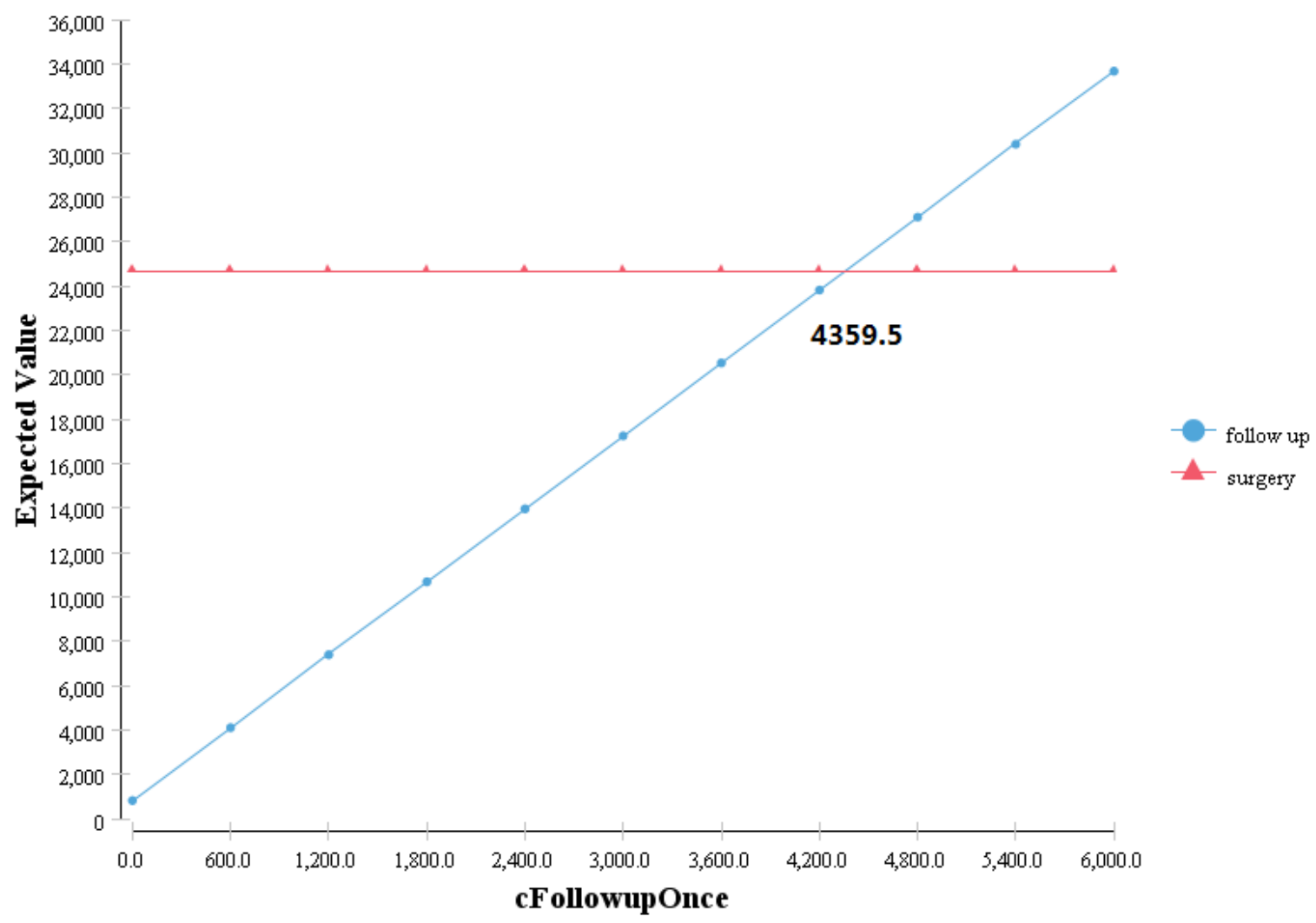

Figure 4

equivalence point of cost of follow-up for once(x-axis: cost of follow-up for once, $y$-axis: expcted value of 2 options; $¥ 4359.5$ for once followup make 2 options equivalent) 
two-way Sensitivity Analysis on dPostSurgery and cFollowupOnce

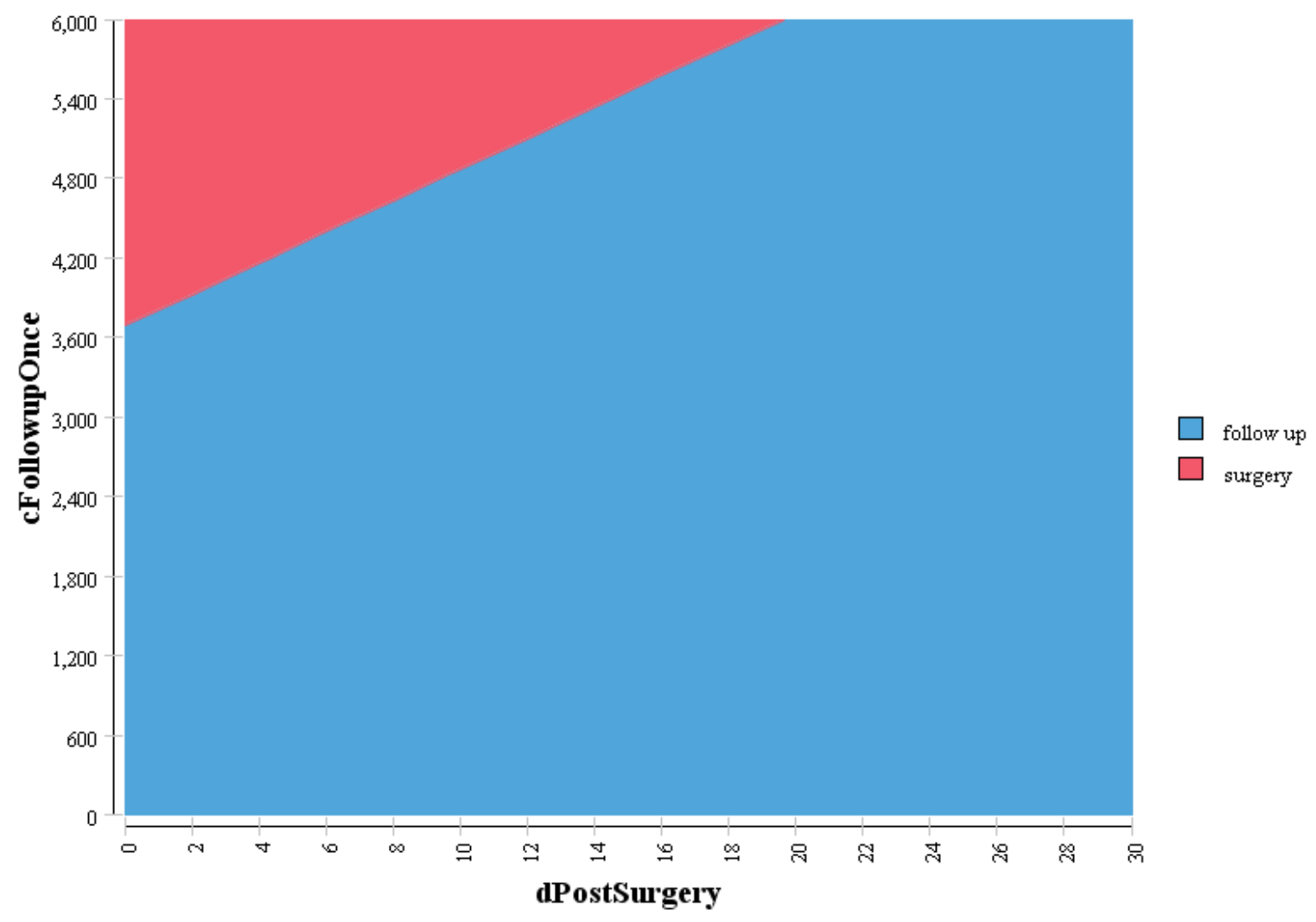

\section{Figure 5}

two-way sensitivity analysis on cost of follow-up for once and length of post-surgery stay(x-axis: length of stay after surgery, $y$-axis: cost of follow-up for once; blue area: follow-up cost less, red area: surgery cost less) 